\title{
CIAC: Cluster Head Induced Affinity based Clustering for Prolonging the Lifetime of Wireless Sensor Network
}

\author{
Sudakshina Dasgupta \\ Govt. College of Engg. and Textile \\ Technology,Serampore,India.
}

\author{
Paramartha Dutta \\ Dept. of Computer and System Science, Visva \\ Bharati University,Santiniketan,India.
}

\begin{abstract}
A WSN is composed of a few hundreds to thousands of spatially distributed autonomous sensor nodes. This large-scale deployment of wireless sensor networks $(W S N s)$ and the need for data aggregation necessitate efficient organization of the network topology for the purpose of balancing the load and prolonging the network lifetime. One of the prior issues in WSNs is how to save the energy consumption for prolonging the network lifetime. Clustering has proven to be an effective approach for organizing the network into a connected hierarchy. The communication or message passing process must be designed to conserve the limited energy resources of the sensors. Sensors are clustered into groups, so that sensors from a cluster communicate information only to cluster heads and then the cluster heads communicate the aggregated information to the processing centre which may save energy. For this purpose, many intelligent innovative techniques are required to improve the energy efficiency and lifetime of the network. In this paper, a new Cluster head Induced Affinity based Clustering (CIAC) algorithm has been proposed that can organize the sensors in a wireless sensor network into clusters through randomized selection of cluster heads and based on the affinity factor of each sensor node induced by the selected cluster head different clusters are formed. The performance of the proposed system is evaluated in terms of energy efficiency and reliability.
\end{abstract}

\section{General Terms}

Cluster head,clustering, LEACH.

\section{Keywords}

Clustering, Wireless sensor network, Cluster head, affinity factor.

\section{INTRODUCTION}

A wireless sensor network (WSN) consists of largely deployed sensor nodes having limited battery power. Sensor nodes of WSN have the capability of self organizing the network [1]. The transmission between the sensor nodes are done through wireless medium. Unique characteristics of a WSN include limited battery power that they are operated with, ability to work in harsh environment, ability to cope with node failure, dynamic network topology and large scale of deployment. Recent years have noticed an increasing interest in using wireless sensor networks (WSNs) in many applications, including environmental monitoring and military field surveillance. In these applications, tiny sensors are deployed and left unattended to continuously report parameters such as temperature pressure, humidity, light, and chemical activity $[2,3,4]$. Data transmitted by these sensors are collected by the base stations. The dense deployment and unattended nature of WSNs make it quite difficult to recharge node batteries. Therefore, energy preservation is a major design goal in these networks. In this case, sensors in different regions of the field can collaborate to aggregate their data and provide more accurate message about their responsible regions $[5,6,7]$. For example, in a habitat monitoring application, the average reported humidity values may be sufficient for the base station. In military fields where chemical activity or radiation is measured, the maximum value may be required to alert the troops. In addition to improving the fidelity of the reported measurements, data aggregation reduces the communication overhead in the network, leading to significant energy savings $[8,9]$. In order to support data aggregation through efficient network organizations, nodes can be partitioned into a number of small groups called clusters [10, 11]. Each cluster has a coordinator, referred to as a cluster head, and a number of sensor member nodes. The cluster head is responsible for the organization of the cluster, data collection and aggregation within the cluster, as well as transmission of the aggregated data to the base station $[12,13]$. Role of Cluster Heads can be rotated to balance the energy utilization by sharing the work load among the sensor nodes. Although formation and maintenance of clusters introduce additional cost but still cluster-based networks have taken much attention of the researchers due to their better performance $[14,15]$. Being a popular clustering protocol, Low Energy Adaptive Clustering Hierarchy (LEACH), has attracted intensive attention of the researchers and has become a well studied and popularly referred baseline. A lot of research work has been carried out to further improve the performance of LEACH protocol [16]

In this work we have proposed an algorithm in which some head nodes are selected (based on the initial number of clusters) from random position over the deployed area. The other sensor nodes join with appropriate head nodes form the initially selected set of cluster heads depending on their distance from the cluster heads and remaining energy of the cluster head which is termed as affinity factor towards the selected cluster head. The concept of determining the affinity value of a node is described in details in Section 3. In this proposed approach set of clusters are formed so that complete 
network coverage can be achieved and with minimum amount of delay data can be transferred from sensor node to the base station via the cluster head. After the formation of cluster, the member nodes send their data to their respective head node, which in turn transmits it to the base station. Once one or more cluster heads become exhausted then alternate head node is selected from the same set of member node of that cluster. Then carry out the transmission without altering the structure of predefined clusters that is induced by defined cluster head. Here the frequent re-clustering and corresponding overhead can be avoided on the total application area. Rest of the paper is organized as follows. Section 2 describes the related work. In section 3 we present our proposed clustering approach Section 4 contains the experimental result and Section $\mathbf{5}$ offers the conclusion of this work.

\section{RELATED WORK}

Many research projects in the last few years have explored clustering in WSN from different perspectives [17]. LEACH is the first clustering algorithm that was proposed for reducing power consumption. In LEACH, the clustering task is rotated among the nodes, based on duration. The operation of LEACH is divided into rounds, where each round begins with a setup phase for cluster formation, followed by a steady state phase, when data transfer to the sink node occur $[16,18]$. In LEACH, a sensor node is elected as the cluster head according to a distributed probabilistic approach. Non cluster nodes decide which cluster to join based on the signal strength. This approach insures lower message overhead, but cannot guarantee that cluster heads are distributed over the entire network uniformly and the entire network is partitioned into clusters of similar size, and the load imbalance over the cluster heads can result in the reduction of network lifetime. LEACH assumes that all nodes have the same amount of energy capacity in each election round which is based on the assumption that being a cluster head results in same energy consumption for every node. Such an assumption is impractical in most application scenarios. LEACH requires source nodes to send data directly to cluster heads. However, if the cluster head is far away from the source nodes, they might expend excessive energy in communication. Furthermore, LEACH requires cluster heads to send their aggregated data to the sink over a single-hop link. However, single-hop transmission may be quite expensive when the sink is far away from the cluster heads. LEACH also makes an assumption that all sensors have enough power to reach the sink if needed which might be infeasible for energy constrained sensor nodes. HEED extends the basic scheme of LEACH by using residual energy and node degree or density as a metric for cluster selection to achieve power balancing $[19,20]$. It operates in multi-hop networks, using an adaptive transmission power in the inter-clustering communication. Both schemes are fully distributed, terminate in constant number of iterations and incur low message overhead. These methods are suitable for prolonging the network lifetime rather than for the entire needs of WSN. In the Linked Cluster Algorithm, a node becomes the cluster head if it has the highest identity among all nodes within one hop of itself or among all nodes within one hop of one of its neighbours [6]. This algorithm was improved by the LCA2 algorithm, which generates a smaller number of clusters. The LCA2 algorithm elects as a cluster head the node with the lowest id among all nodes that are neither a cluster head nor are within 1-hop of the already chosen cluster heads. The algorithm proposed in [6], chooses the node with highest degree among its 1-hop neighbours as a cluster head. All of the algorithms which generate 1-hop clusters, require synchronized clocks and have a complexity of $\mathrm{O}(\mathrm{n})$. This makes them suitable only for networks with a small number of nodes. The Max-Min dcluster Algorithm proposed in generates d-hop clusters with a run-time of $\mathrm{O}(\mathrm{d})$ rounds. This algorithm achieves better load balancing among the cluster heads, generates fewer clusters than the LCA and LCA2 algorithms and does not need clock synchronization. In CEFL (Clusterhead Election Using Fuzzy Logic) algorithm, fuzzy logic method is adopted to select the cluster head [21, 22, 23]. Most of these clustering methods rely on synchronization. PEGASIS still requires dynamic topology adjustment since a sensor node needs to know about the energy status of its neighbours in order to know where to route its data [24]. Such topology adjustment can introduce significant overhead, especially for highly utilized networks. Moreover, PEGASIS assumes that each sensor node is able to communicate with the base station directly. In practical cases, sensor nodes use multihop communication to reach the BS. Also, PEGASIS assumes that all nodes maintain a complete database of the location of all other nodes in the network. In addition, PEGASIS assumes that all sensor nodes have the same level of energy and are likely to die at the same time. Note also that PEGASIS introduces an excessive delay for distant nodes on the chain. In addition, the single leader can become a bottleneck. Finally, although in most scenarios sensors will be fixed or immobile as assumed in PEGASIS, some sensors may be allowed to move and hence affect the protocol functionality. In game playing approach of cluster-head selection for wireless sensor networks, the payoff matrix plays a vital role in the Game playing approach to select the cluster heads in the network clusters and one can change the final game result only by changing the payoff matrix [25]. In [26] the author has proposed an election algorithm to select the $\mathrm{CHs}$ in a democratic fashion by the nodes in the network. In the proposed democratic election procedure each node will have the right to vote to select a leader within its cluster using a voting procedure and after that the elected node becomes the cluster head of that cluster. In [27] the author has present a new approach of cluster head selection strategy embedded with the traditional Fuzzy c-means algorithm with minimum distance and maximum residual energy criteria satisfied. Fuzzy C-means is a method of clustering which algorithm allows one piece of sensor node to belong to two or more clusters. With fuzzy Cmeans, the centroid of a cluster is computed as being the mean of all sensor points, weighted by their degree of belonging to the cluster. The degree of being in a certain cluster is related to the inverse of the distance to the cluster. By iteratively updating the cluster centres and the membership grades for each sensor node is updated. The fuzzy c-means algorithm iteratively moves the cluster centres to the right location within a set of nodes.

\section{PROPOSED WORK}

In most of the approaches that are considered in literature the number of clusters was fixed. The cluster heads were selected based on some selection criteria. In the proposed 
work, an algorithm has been designed in which some head nodes are selected randomly over the deployed area. The other sensor nodes join with appropriate head node form the initially selected set of cluster heads depending on their affinity factor which is defined by the ratio of the residual energy and the minimum distance from that node to the base station via selected head node as shown in Figure 1. In this approach a set of selected cluster heads induce a set of clusters so that complete network coverage can be achieved and with minimum amount of delay data can be transferred from sensor node to the base station via the cluster head. After the formation of clusters, the member nodes send their data to their respective head node, which in turn transmits it to the base station. Once one or more cluster heads become exhausted then an alternative member node can take the responsibility of that cluster depending on their cumulative affinity value over all nodes on that cluster and the remaining energy of that node to avoid the reclustering on the entire application area unnecessarily, which may lead to a very cost effective approach. Here Figure 2 represent the snapshot of the simulation carried out.

\begin{tabular}{|c|c|c|c|c|c|c|c|}
\hline & $\mathrm{CH}_{1}$ & $\mathrm{CH}_{2}$ & $\mathrm{CH}_{3}$ & $\mathrm{CH}_{4}$ & $\mathrm{CH}_{5}$ & $\ldots$ & $\mathrm{CH}_{\mathrm{k}}$ \\
\hline$N_{1}$ & $\operatorname{aff}(1,1)$ & $\operatorname{aff}(1,2)$ & $\operatorname{aff}(1,3)$ & $\operatorname{aff}(1,4)$ & $\operatorname{aff}(1,5)$ & $\ldots$ & $\operatorname{aff}(1, \mathrm{k})$ \\
\hline $\mathrm{N}_{2}$ & $\operatorname{aff}(2,1)$ & $\operatorname{aff}(2,2)$ & $\operatorname{aff}(2,3)$ & $\operatorname{aff}(2,4)$ & $\operatorname{aff}(2,5)$ & $\ldots$ & $\operatorname{aff}(2, \mathrm{k})$ \\
\hline$N_{3}$ & $\operatorname{aff}(3,1)$ & $\operatorname{aff}(3,2)$ & $\operatorname{aff}(3,3)$ & $\operatorname{aff}(3,4)$ & $\operatorname{aff}(3,5)$ & $\ldots$ & $\operatorname{aff}(3, \mathrm{k})$ \\
\hline $\mathbf{N}_{4}$ & $\operatorname{aff}(4,1)$ & $\operatorname{aff}(4,2)$ & $\operatorname{aff}(4,3)$ & $\operatorname{aff}(4,4)$ & $\operatorname{aff}(4,5)$ & & $\operatorname{aff}(4, \mathrm{k})$ \\
\hline$N_{5}$ & $\operatorname{aff}(5,1)$ & $\operatorname{aff}(5,2)$ & $\operatorname{aff}(5,3)$ & $\operatorname{aff}(5,4)$ & $\operatorname{aff}(5,5)$ & $\ldots$ & $\operatorname{aff}(5, \mathrm{k})$ \\
\hline .. & $\ldots$ & $\ldots$ & $\ldots$ & $\ldots$ & $\ldots$ & $\ldots$ & $\ldots$ \\
\hline$N_{n}$ & $\operatorname{aff}(\mathrm{n}, 1)$ & $\operatorname{aff}(\mathrm{n}, 2)$ & $\operatorname{aff}(\mathrm{n}, 3)$ & $\operatorname{aff}(\mathrm{n}, 4)$ & $\operatorname{aff}(\mathrm{n}, 5)$ & $\ldots$ & aff(n,k) \\
\hline
\end{tabular}

Fig 1. An Affinity Matrix with $\mathrm{n}$ nodes and $\mathrm{k}$ cluster heads.

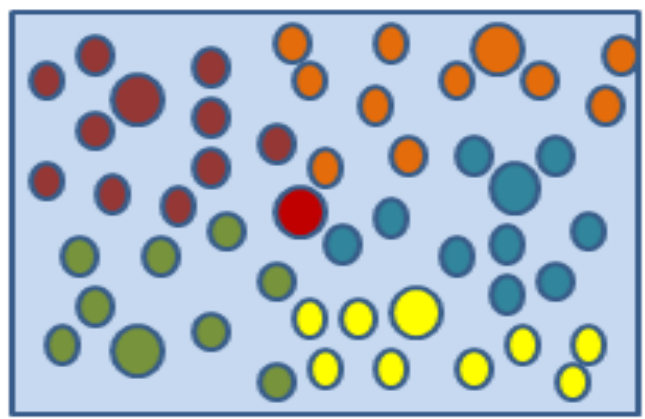

Fig 2. Snapshot of the simulation with number of sensor nodes, base station and cluster heads.

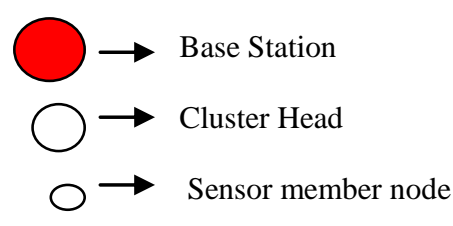

The proposed CIAC algorithm is described as follows:

\subsection{CIAC Algorithm for Cluster formation and Cluster Head rotation.}

Step 1: Initialize residual energy of each node and fix the position of the nodes. Repeat steps 2 through 7 until battery power of the nodes in the network get exhausted.
Step 2: Randomly select $k$ number of cluster heads $C 1$ $, C 2, C 3, \ldots C k$ from $n(\mathrm{n}>>\mathrm{k})$ number of nodes over an application area.

Step 3: In order to maintain the uniformity of formed clusters in terms load balancing in the network, Affinity matrix is computed based on the residual energy of each node and sum of the distance from any of the member node to the cluster head node and from the cluster head node to the base station. Each entry of the affinity matrix can be calculated by the

$$
\text { Affinity factor }\left(\operatorname{aff}\left(x, c_{i}\right)\right)=\frac{R(x)}{d\left(x, c_{i}\right)+d\left(c_{i}, B S\right)}
$$

where $R(x)$ is the Residual energy of member node $x, c_{i}$ is the $i^{\text {th }}$ cluster Head node, $\mathrm{d}\left(\mathrm{x}, \mathrm{c}_{\mathrm{i}}\right)$ is the distance between member node and corresponding head node and $\mathrm{d}\left(\mathrm{c}_{\mathrm{i}}, \mathrm{BS}\right)$ is the distance between head node and base station. Here $1 \leq \mathrm{i} \leq \mathrm{k}$.

Step 4: From this affinity matrix find the $(\mathrm{j}, \mathrm{i})$ position where the position defines the maximum affinity value of the respective cluster head where a $\mathrm{j}^{\text {th }}$ non head node is associated with $\mathrm{i}^{\text {th }}$ cluster head. Here $1 \leq \mathrm{j} \leq \mathrm{n}-\mathrm{k}$ and $1 \leq \mathrm{i}$ $\leq \mathrm{k}$. From the result of affinity matrix, different clusters are formed. Then calculate summation of the matrix $S$ for $\mathrm{k}$ number of cluster head

$$
S_{i}=\sum_{\mathrm{i}=1}^{\mathrm{k}} \sum_{\mathrm{j}=1}^{\mathrm{n}-\mathrm{k}} \frac{R\left(x_{j}\right)}{d\left(x_{j}, c_{i}\right)+d\left(c_{i}, B S\right)}
$$

where sum of values of all the column is equivalent to sum of values of all the rows. Compute a table for each of the cluster consisting of the following information cluster id, node id, coordinate position and residual energy.

Step 5: Allow data transmission for $\Delta t$ time.

Step 6: Consult the table information of each cluster in terms of residual energy to ascertain the change of head node within a cluster (if needed) without affecting the cluster topology itself.

Step 6.1: For alternative head selection if the table information gives another node having higher energy after $\Delta \mathrm{t}$ time data transmission then if the total affinity value (cumulative over all nodes within that cluster) is less than that of the previously defined head's cumulative affinity and at that time previously head has energy (greater than the threshold) then allow the previously defined head to continue the responsibility of Cluster head .

Else

If the total affinity value is not greater than the predefined head's total affinity but the energy is less than the threshold of this predefined head then select the new one considering new affinity value.

Step 7: Carry out performance analysis altering the value of $\Delta \mathrm{t}$ time until the clusters become exhausted. Step 8: stop.

\section{EXPERIMENTAL RESULT}


In the proposed work, the experiment has been carried over with 100 sensor nodes deployed randomly over a simulation area. The head nodes are selected arbitrarily from the deployed sensor nodes. The other sensor nodes are joined with the appropriate head nodes depending on their affinity factor which is determined by the ratio of the residual energy and the minimum distance from that node to the base station through the respective head nodes. The inter-cluster communication will happen only through cluster heads of the respective clusters. The base station is positioned at $(150,150)$. A node is considered to be a dead node if its energy level is zero and the threshold is defined as $10 \%$ of its initial energy of the respective nodes. The performance of LEACH algorithm is compared with the proposed algorithm in terms of communication overhead, the number of communication rounds, total amount of energy dissipated in the system over time and the number of dead nodes in the system over time using simulator based on JAVA. For a node in the sensing state, packets are generated at a constant rate of 1 packet/sec. Considering the fact that once the sensors are deployed in the system they are fixed and mobility is restrained. The Euclidian distance is calculated between the sensors within a cluster. The system is free of contention and error considered here and hence the sensors need not retransmit data. To compute energy consumption for each transmission sending and receiving, the radio energy dissipation model is used. The energy used to transmit $q$ bits of data at a distance $d$ for each sensor node is

$$
\operatorname{ET} T_{X}(q, d)=q E E l e c+q \in f s d^{2}
$$

The energy used to receive data for each node is $E R x(q)=q E E l e c, \quad$ where EElec is electronic energy and $\ell f s d^{2}$ is power loss of free space. In these experiments, each node begins with an initial energy of 0.5 joule and unlimited amount of data can be sent to the Base station via cluster head. In the following table, the parameters for experiments are summarized below .

\begin{tabular}{|l|l|}
\hline \multicolumn{2}{|c|}{ Experiment Setup } \\
\hline Parameters & Value \\
\hline Network Size & $300 \mathrm{X} 300$ \\
\hline Number of sensors & 100 \\
\hline Base station location & $(150,150)$ \\
\hline Packet generating rate & $1 \mathrm{packet} / \mathrm{sec}$ \\
\hline EElec & $50 \mathrm{nj} / \mathrm{bits}$ \\
\hline$\epsilon f s$ & $10 \mathrm{nj} / \mathrm{bits} / \mathrm{m}^{2}$ \\
\hline Initial energy & $0.5 \mathrm{jules}$ \\
\hline $\begin{array}{l}\text { Data packet size(nodes to } \\
\text { cluster head) }\end{array}$ & $550 \mathrm{bytes}$ \\
\hline $\begin{array}{l}\text { Data packet size(head to } \\
\text { base station) }\end{array}$ & $600 \mathrm{bytes}$ \\
\hline
\end{tabular}

\section{Table 1. Simulation Parameters}

In these experiments, each node is assigned with an initial energy of 0.5 joule and unlimited amount of data can be sent to the Base station via cluster head. In figure 3 the experiment has been carried out to justify the changes of cluster heads with time. At first the time interval $\Delta t$ has been taken very small and the changes of cluster heads are observed at equal time interval $\Delta \mathrm{t}$. It can be observed that at the beginning of the process the number of cluster head rotation was very small. As the time grows due to energy dissipation of the nodes, specially cluster head it is required to change the cluster heads more frequently. The same experiment has been carried out considering variable time interval $\Delta \mathrm{t}$ and figure 4 shows the experimental result, which is more or less similar to figure 3 . In figure 5 the experiment has been carried out to find out the number of dead nodes with time, which shows the efficiency of the proposed algorithm in comparison to existing LEACH and Fuzzy $\mathrm{C}$-means algorithm in terms of the usage of the node energy in an efficient way. Figure 5 clearly shows that the proposed algorithm is better than the existing LEACH and fuzzy C-means algorithm as it uses the network energy in an efficient manner. The cluster heads are not going to be rotated in each round in contrast with existing LEACH algorithm rather the cluster heads are going to change as and when required in the proposed algorithm. With Fuzzy C-means, the centroid of a cluster is computed as being the mean of all sensor points, weighted by their degree of belonging to the cluster. The degree of being in a certain cluster is related to the inverse of the distance to the cluster. By iteratively updating the cluster centres and the membership grades for each sensor node is updated. The Fuzzy C-means algorithm iteratively moves the cluster centres to the right location within a set of nodes. In the proposed algorithm as the respective cluster heads need not necessarily be the centroid nodes therefore it uses the network energy in an efficient way. Figure 6 also justifies the claim as number of alive nodes is more in the case of the proposed algorithm in contrast to existing LEACH and Fuzzy C-means algorithm. Hence the proposed algorithm is more energy efficient and can increase the network lifetime in the case of Wireless Sensor Network.

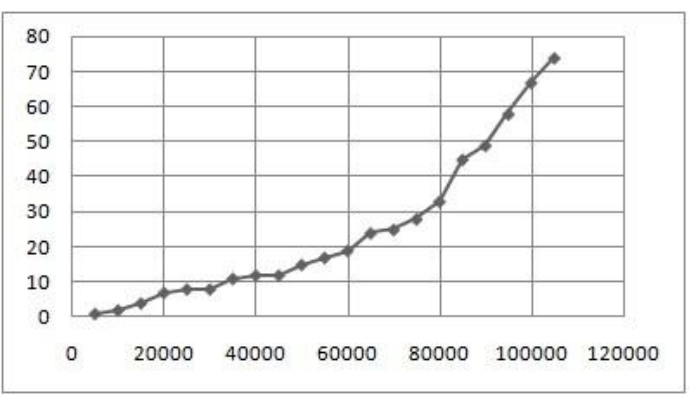

Fig 3. changes of cluster heads with time $\Delta t$ (fixed interval)

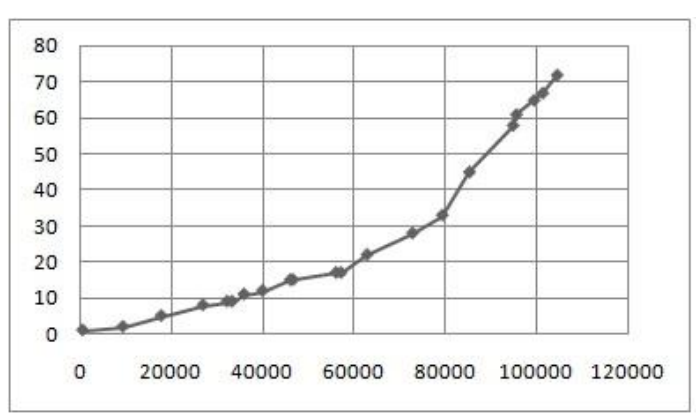

Fig 4. changes of cluster heads with time $\Delta t$ (variable interval) 


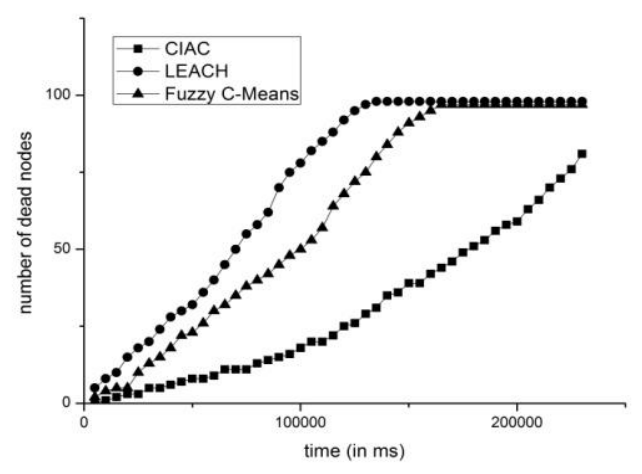

Fig 5. Graph showing Number of dead nodes with time

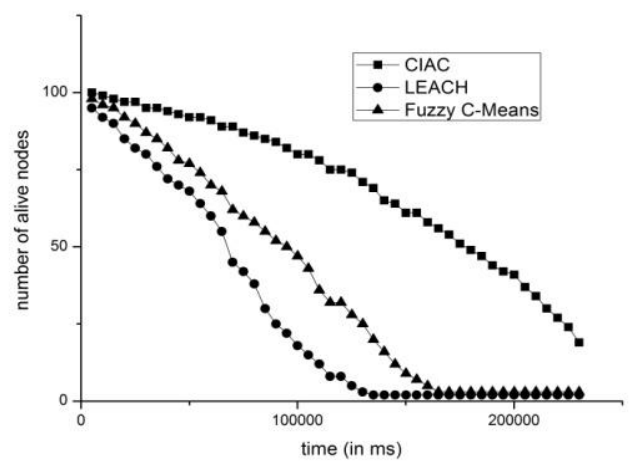

Fig 6. Graph showing Number of alive nodes with time

\section{Conclusion}

In this paper an alternative method for clustering the network is developed by the random selection of cluster head in first step. Then the selected cluster head induces the other member nodes by the affinity factor where the nodes are distributed over the projected domain and form the clusters. The basis of this method is the intelligent formation of clusters in the network in an balanced way to enhance the network lifetime. This approach is better than the existing LEACH and Fuzzy C-means algorithm in terms of network lifetime. The cluster head rotation is one of the main concerns in this context. The alternate cluster head selection when required can be dynamically adjusted. The new affinity factor is calculated which in turn affect the cluster head selection process. Here it has been assumed that all the nodes are fixed at location and not mobile. We have also assumed that the number of clusters is fixed, nodes are identical and have the same initial energy. But in many applications these assumptions may not be realistic. As in our algorithm cluster heads do not change in each round it can preserve the maximum network coverage. In our approach we have considered the residual energy of a node in the calculation of affinity value of a node that leads to increase of network life time.

\section{REFERENCES}

[1] I.F. Akyildiz, W. Su, Y. Sankara subramniam, and E. Cayirei, Aug.2002. A survey on sensor networks , IEEE Commun Mag., vol.40, no.8, pp.102-114.

[2] C-Y. Chong, S.P. Kumar, 2003. Sensor Networks: evolution, opportunities and challenges, Proceeding of IEEE 2003,vol. 91(8) pp. 1247-1256.

[3] H. Wang, et al. April 2003. Target classification and localization in habitat monitoring, Proceedings of IEEE International Conference on Acoustics, Speech and Signal Processing Hong Kong china.,Vol. 4, pp. 844-847.

[4] D.E. Goldberg, 1989. Genetic Algorithms in Search, optimization and Machine Learning, Addison Wesley, New York, .

[5] A.B. McDonald, T. Znati, 1999. A mobility based framework for adaptive clustering in wireless adhoc networks", IEEE Journal on Selected Areas in Communications 17 (8) ,pp. 1466-1487.

[6] S. Bandyopadhyay , E. Coyle, 2003. An energy efficient hierarchical clustering algorithm for wireless sensor networks, Proceedings of the 22nd Annual Joint Conference of the IEEE Computer and Communications Societies, San Francisco, California ,Vol. 3, pp. 1713-1723.

[7] W. B. Heinzelman , A. P. Chandrakasan , H. Balakrishnan, 2002. Application specific protocol architecture for wireless microsensor networks,IEEE Transactions on Wireless Networking, pp. 660-670.

[8] Li Qing, Qingxin Zhu,2006. Mingwen Wang Design of a distributed energy-efficient clustering algorithm for heterogeneous wireless sensor networks, Computer Communications, 29, pp. 2230-2237.

[9] D. Turgut, S. K. Das, R. Elmasri, and B. Turgut, Optimizing clustering algorithm in mobile adhoc networks using genetic algorithmic approach, Proceedings of the Global Telecommunications conference (GLOBECOM), 2002, IEEE ,Vol. 1, pp. 62-66.

[10] G. Wang ,H. Dai ,L. Wu and B.Xiong,2009.The clustering algorithm of wireless sensor networks based on multihop between clusters, Computer Science and Information Engineering, vol. 3, pp 177-181.

[11] J.D. Yu, K.T. Kim ,B.Y. Jung and H,Y. Youn,2009.An Energy Efficient Chain-Based Clustering Routing Protocol for Wireless Sensor Networks ,Advanced Information Networking and Application Workshop , pp. 383-388.

[12] Jamshid Shanbehzadeh ,Saeed Mehrjoo, Abdolhossein Sarrafzadeh ,2011. An Intelligent Energy Efficient Clustering in Wireless Sensor Networks, Proceedings of the International Multi conference of Engineers and computer Scientists , Vol 1, IMECS arch 16-18,Hong Kong 
[13] M.J. Handy ,M. Haase, and D. Timmermann, September 2002.Low energy adaptive clustering hierarchy with deterministic cluster-head selection, Proceedings of the 4th International Workshop on Mobile and Wireless Communications Network ,pp.9-11.

[14] A. John Wiley, D. Minoli and T. Znati, 2007. Wireless Sensor Networks Technology, Protocols and Applications, Inc. ,Publication .

[15] J. Yu, W. Liu, J. Song and B. Cao, 2008. EEMR: An energy-efficient multihop routing protocl for wireless sensor networks, Computer System and Applications(AICCSA), pp.291-298.

[16] W. Heizelman, A Chandrakasan, H. Balakrishman,2000.Energy-efficient routing protocols for wireless micro sensor networks, Proc 33rd Hawaii international conferences on system sciences (HICS'00),2000,pp 223-232.

[17] Rong Ding ,Bing Yang, Jiawei Wang,September 2009.Soft Threshold based Cluster-head selection algorithm for Wireless Sensor Networks,3rd International Conference on Sensor Technologies and Applications ,pp 526-530.

[18] A. Khademzadeh and K. Khamfroush , April 2009.Lifetime Increase for Wireless Sensor Networks using Cluster-Based Routing,International Association of Computer Science and Information Technology-Spring Conference(IACSITSC),pp. 1418.

[19] O. Younis, S. Fahmy,2004. HEED: A Hybrid, Energy-Efficient, Distributed clustering approach for Ad Hoc sensor networks,IEEE Transactions on Mobile Computing, vol. 3(4),pp.366-379.

[20] W.W. Huang, M. Yu ,L. Q. Xiong and J. Wen ,Dec 2008.Energy-Efficient Hierarchical Routing
Protocol for Wireless Sensor Networks ,Computational Intelligence and Indrustrial Application (PACIIA), vol.1, pp. 640-644.

[21] J.M. Mandel ,1995.,Fuzzy Logic System for Engineering: A Tutorial, Proc of the IEEE 83(3), pp. 345-377.

[22] Gupta I., Riordan D., and Sampalli S, May 2012.Cluster-head election using fuzzy logic for wireless sensor networks proceeding of the 3rd Annual Communication Networks and services Research Conference Halifax, pp.255-260.

[23] J. Anno, L. Barolli, F. Xhafa and A. Durresi,2007.A Cluster-head Selection Method for Wireless Sensor Networks Based on Fuzzy logic, proceedings of IEEE Tencon-, pp. 1-4

[24] S.Lindsey and C. S. Raghavendra, March 2002. PEGASIS: Power-efficient gathering in sensor information systems, Proceedings of the IEEE Aerospace Conference, Vol. 3, pp. 1125-1130.

[25] Sudakshina Dasgupta, Paramartha Dutta ,2013 . A Novel Game Theoretic Approach for Cluster Head Selection in WSN ,International journal of Innovative Technology and Exploring Engineering(IJITEE), ISSN:2278-3075,Vol-2,Issue3 , pp 40-43.

[26] Sudakshina Dasgupta, Paramartha Dutta, September 2012.A Democratic Head Election Strategy for Clustering in Wireless Sensor Network .International Journal of Computer Applications (0975 -8887), Volume 54- No.16, pp. 32-36.

[27] Sudakshina Dasgupta , Paramartha Dutta,2011.An Improved Leach approach for Head selection Strategy in a Fuzzy-C Means induced Clustering of wireless Sensor Network, IEMCON 2011 pp. 203208. 